\title{
Doctorado en Estudios Interculturales: un análisis de pertinencia y factibilidad para el Modelo de Universidad Comunitaria Intercultural
}

\author{
Intercultural Studies Doctorate: A relevance and feasibility \\ analysis for the Intercultural Community University Model
}

\author{
William Oswaldo Flores López ${ }^{1}$ \\ Letisia Castillo Gómez ${ }^{2}$
}

\section{Resumen}

Esta investigación ha analizado la pertinencia y factibilidad de un programa de doctorado en estudios interculturales en la Universidad Comunitaria Intercultural, de la Universidad de las Regiones Autónomas de la Costa Caribe Nicaragüense (URACCAN). Se aplicaron métodos y técnicas de los paradigmas cualitativo y cuantitativo, sustentados en un diseño descriptivo, los datos fueron resultado de una entrevista semi-estructurada a hombres y mujeres de la URACCAN y a futuros empleadores de investigadores con el nivel de doctorado; además, se revisó documentación de políticas, normativas, artículos científicos y publicaciones en diarios de comunicación digital. El análisis se desarrolló utilizando el paquete estadístico para las Ciencias Sociales y una matriz de categorización. Los principales resultados reflejaron que existe demanda de formación de talentos humanos en el grado de doctorado para alcanzar los niveles de competitividad en investigación, desarrollo e innovación; así como una demanda por parte de profesionales con grado de maestría por realizar estudios doctorales. Asimismo, se cuenta con recursos físicos, financieros y humanos para desarrollar el programa de doctorado. Todo lo anterior, nos permite avalar la necesidad de formar doctores en estudios interculturales que respondan a las necesidades y aspiraciones de los pueblos de la Costa Caribe y Ab'ya Yala.

Palabras clave: Pertinencia; factibilidad; doctorado; interculturalidad.

\section{Abstract}

This research has analyzed the relevance and feasibility of a doctoral program in intercultural studies at the University of the Autonomous Regions of the Nicaraguan Caribbean Coast (URACCAN) the Intercultural Community University. Qualitative and quantitative methods and techniques were applied, based on a descriptive design, the data were the result of a semi-structured interview to URACCAN men and

1 Doctor en Educación. Profesor Investigador de la Universidad de las Regiones Autónomas de la Costa Caribe Nicaragüense. Correo: william.flores@uraccan.edu.ni ORCID: http://orcid.org/oooo-ooo2-1016-1620

2 Candidata Doctoral en Educación Superior. Directora Académica General de la Universidad de las Regiones Autónomas de la Costa Caribe Nicaragüense. Correo: direccion.academica@uraccan.edu.ni ORCID: https://orcid.org/oooo-0oo2-1585-1049 


\section{EDUCACIÓN}

women and to future employers of researchers with the doctorate level; In addition, documentation of policies, regulations, scientific articles and publications in digital communication journals were reviewed. The analysis was developed using the statistical package for the social sciences and a categorization matrix. The main results reflected that there is a demand for the training of human talents in the doctorate degree to reach the research competitiveness, development and innovation levels; as well as a demand from the professionals with a master's degree for carrying out doctoral studies. Likewise, there are physical, financial and human resources to develop the doctoral program. All of the above allows us to endorse the need to train intercultural studies doctors that respond to the needs and aspirations of the Caribbean Coast and Ab'ya Yala peoples.

Keywords: Relevance; Feasibility; Doctoral Program; Interculturality

\section{Introducción}

Un programa de doctorado es ofrecer las perspectivas que concreten el desarrollo de las habilidades profesionales, académicas y humanas. Permite dar respuesta a las demandas de formación de talentos humanos en investigación, para la generación de conocimientos y las relaciones entre sociedad, praxis de ciencia y técnica para plantear soluciones a los problemas de las comunidades apreciando los diferentes escenarios socioculturales de la Costa Caribe de Nicaragua, el país y los desafíos de la diversidad cultural en un mundo globalizado que busca comprender, explicar y mejorar las interacciones de los pueblos, especialmente en contextos pluriculturales de la América Latina y el Caribe.

Es así que brinda la preparación para la investigación y la innovación, que genere aportes originales significativos al acervo de conocimientos en un área específica. Exige aprobar un número establecido de actividades académicas; demostrar la superación de distintos niveles de complejidad en el saber que permitan avanzar y desplazar las fronteras de un campo del conocimiento y presentar un trabajo original de investigación sobre un tema relacionado con el campo científico, tecnológico o artístico, de acuerdo con la naturaleza del programa: tesis, disertación doctoral o publicación de artículos en revistas indexadas (Abreu, Cruz \& Martos, 2014).

Desde esta perspectiva, la presente investigación tiene el propósito de analizar la pertinencia y factibilidad de un programa doctoral en estudios interculturales. Mediante revisión de la información sobre la tendencia y datos estadísticos sobre el empleo de investigadores de Nicaragua; se ha entrevistado a empleadores sobre las expectativas y competencias de los futuros doctores en estudios interculturales; asimismo a profesionales sobre la tendencia y demanda de estudios doctorales; y se describe la factibilidad de la apertura de un programa de doctorado en estudios interculturales. 


\section{Revisión de literatura}

En la formación doctoral se suelen distinguir dos etapas: la primera de dominio del campo, le brinda al estudiante conocimiento amplio y profundo para que sea capaz de identificar potenciales problemas de investigación, plantear hipótesis y determinar los mejores métodos y técnicas para probarlas o descartarlas. Esta primera etapa puede coincidir con los estudios de la maestría de investigación. La segunda etapa, consiste en llevar a la práctica el proyecto de investigación, analizar los resultados y obtener conclusiones válidas, que constituyan aportaciones relevantes al campo del conocimiento (Abreu, Cruz \& Martos, 2014).

En este sentido, los estudios de pertinencia de programas académicos apuntan al análisis de la capacidad de las Instituciones de Educación Superior para responder a las demandas de la sociedad actual. Según Castillo et al (2013) "la evaluación de la pertinencia estudia aspectos referentes a las prioridades establecidas por los planes de desarrollo; estudios de oferta y demanda educativa; estudios de competencia y de formación integral del estudiantado, así como de los mecanismos de producción y distribución del conocimiento" (p. 49). Para la UNESCO (1998) la pertinencia en Educación Superior se considera primordialmente en función de su cometido y su puesto en la sociedad de sus funciones con respecto a la enseñanza, la investigación y los servicios conexos y de sus nexos con el mundo de trabajo en sentido amplio con el Estado y la financiación pública y sus interacciones con otros niveles y formas de educación (p. 19).

Además, la pertinencia está vinculada a una de las principales características que tiene el nuevo contexto de producir conocimientos, énfasis en valorar el entorno en el cual están insertas las instituciones de investigación, por lo tanto, la necesidad de un estrecho acercamiento entre los que producen y entre los que se apropian del conocimiento. Por un lado, los que se apropian, o sea los usuarios del conocimiento, son no solamente los estudiantes o usuarios internos, sino las comunidades en la que están implantadas las instituciones, y también, de manera importante, los otros niveles del sistema educativo (García-Guadilla, 1997, pp. 64-65). En definitiva, la pertinencia se consigue efectivamente en la participación de la institución educativa, a través de sus actores, en la vida social, económica y cultural de la sociedad, en la cual ella se inserta, en especial con la gente en su entorno, sin jamás perder la perspectiva de la universalidad (Sobrinho et al., 2008).

Con relación a la factibilidad, se define como las posibilidades de las instituciones académicas para la ejecución de programas académicos de alto nivel en términos de recursos financieros, físicos, infraestructura, talentos humanos y materiales, con lo cual se garantice el funcionamiento adecuado del futuro programa académico. Es importante agregar a esta definición de factibilidad la necesidad de que la propuesta curricular tenga un marco legal que la fundamente (Arrieta de Meza y Meza-Cepeda, 
2014). Además, es importante mencionar que la factibilidad y la pertinencia tiene una estrecha relación, porque el estudio de factibilidad mide el éxito de un programa académico que se desarrolla, y la pertinencia es unos de los criterios que se emplea en la política educativa con el propósito de que las instituciones de la Educación Superior, sean agentes de cambios que coadyuven al mejoramiento del contexto social donde implementan acciones educativas.

\section{Materiales y métodos}

\section{Metodología y diseño de investigación}

El análisis sobre la pertinencia y factibilidad de un programa de Doctorado en Estudios Interculturales se realizó en la Universidad de las Regiones Autónomas de la Costa Caribe Nicaragüense durante el segundo semestre del 2017. Es por ella, que la presente investigación es de carácter cuantitativa porque ha guiado el tratamiento de los datos a través de la categorización y descripción de las propiedades, características y perfiles de las personas, grupos, comunidades, procesos y objetos que se han sometido a análisis (Hernández, Fernández, \& Baptista, 2010). Además, incorpora elementos de la metodología de la investigación cualitativa porque es una actividad sistemática orientada a la comprensión en profundidad de fenómenos educativos y sociales, a la transformación de prácticas y escenarios socioeducativos, a la toma de decisiones y también hacia el descubrimiento y desarrollo de un cuerpo organizado de conocimiento (Bisquerra, 2009). El diseño de esta investigación es descriptivo, porque constituye una opción de investigación cuantitativa que trata de realizar descripciones precisas y muy cuidadosas respecto al fenómeno educativo a investigar (Bisquerra, 2012, p. 197).

\section{Participantes}

En el estudio de expectativas de los empleadores sobre las competencias de los futuros doctores en estudios interculturales, se entrevistaron a organizaciones educativas e instituciones que gerencia la Educación Superior en el ámbito nacional e internacional. En cambio, el estudio sobre la tendencia y demanda de formación se llevó a cabo con una muestra de 52 participantes con el grado de maestría de los grupos étnicos miskitu, mayangna, creole y mestizo de la URACCAN, pertenecientes a comunidades de la Costa Caribe de Nicaragua. 


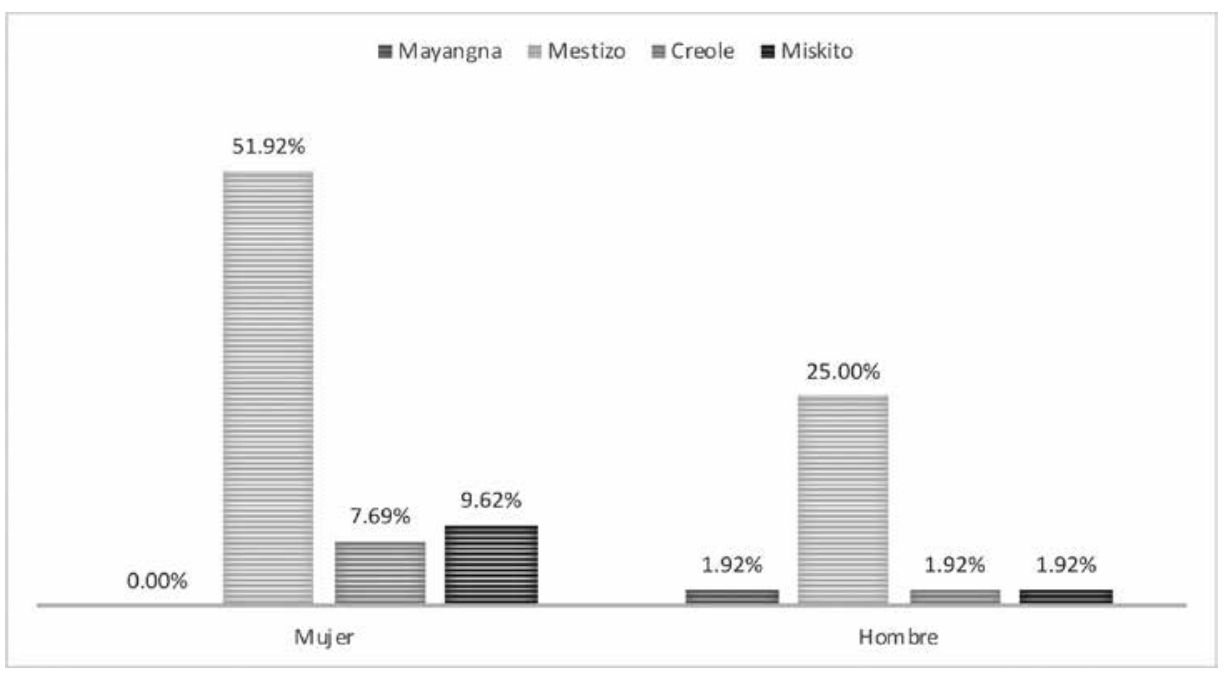

Figura 1: Distribución de los participantes en función de las variables género y etnia.

El 69.21\% de los participantes son mujeres, frente al 30.79\% que son hombres. Respecto a su edad media se sitúa entre 40 , encontrándose al $57 \%$ de los participantes en el intervalo de edad entre 35-45 años. En referencia a la variable etnia: el 11.54\% son indígenas miskitus; el 1.92\% son indígenas mayangnas; el 9.62\% creole (afrodescendientes) y el $76.92 \%$ mestizos. Otra característica de los participantes es sobre el grado de Maestría que han realizado en sus estudios de postgrado. Véase en el cuadro 1:

Cuadro 1: Distribución de los participantes por sus estudios de maestría y género

\begin{tabular}{|l|l|l|l|l|l|}
\hline$N^{\prime}$ & \multicolumn{1}{|c|}{ Estudios de Maestría } & Mujeres & Hombres & Total & \multicolumn{1}{c|}{$\%$} \\
\hline 1 & Antropología Social & 5 & 0 & 5 & $10 \%$ \\
\hline 2 & Docencia Universitaria & 15 & 6 & 21 & $40 \%$ \\
\hline 3 & Agronegocios & 2 & 0 & 2 & $4 \%$ \\
\hline 4 & Agro Forestaría Tropical & 0 & 2 & 2 & $4 \%$ \\
\hline 5 & Salud Intercultural & 2 & 0 & 2 & $4 \%$ \\
\hline 6 & Planificación Estratégica & 0 & 1 & 1 & $2 \%$ \\
\hline 7 & Comunicación Intercultural & 0 & 2 & 2 & $4 \%$ \\
\hline 8 & Administración Pública y Desarrollo Rural & 0 & 1 & 1 & $2 \%$ \\
\hline 9 & Desarrollo Rural & 0 & 1 & 1 & $2 \%$ \\
\hline 10 & Educación Intercultural Multilingüe & 3 & 0 & 3 & $6 \%$ \\
\hline & $\begin{array}{l}\text { Metodología, Evaluación y Diagnostico de la Educación } \\
\text { Superior }\end{array}$ & 1 & 0 & 1 & $2 \%$ \\
\hline 12 & Formulación y Evaluación de Proyectos & 0 & 1 & 1 & $2 \%$ \\
\hline
\end{tabular}




\begin{tabular}{|l|l|l|l|l|l|}
\hline No & \multicolumn{1}{|c|}{ Estudios de Maestría } & Mujeres & Hombres & Total & \multicolumn{1}{c|}{$\%$} \\
\hline 13 & Gerencia Estratégica & 2 & 0 & 2 & $4 \%$ \\
\hline 14 & Medio Ambiente y Gestión de Cuencas & 2 & 0 & 2 & $4 \%$ \\
\hline 15 & $\begin{array}{l}\text { Desarrollo con Identidad: Gobernabilidad y Manejo de } \\
\text { Bosques }\end{array}$ & 0 & 1 & 1 & $2 \%$ \\
\hline 16 & Educación Rural & 1 & 1 & 2 & $4 \%$ \\
\hline 17 & Dirección Estratégica e Ingeniería de Software & 2 & 0 & 2 & $4 \%$ \\
\hline 18 & Administración de Recursos Humanos & 1 & 0 & 1 & $2 \%$ \\
\hline & Total & 36 & 16 & 52 & $100 \%$ \\
\hline
\end{tabular}

Para concluir, indicar que el tipo de muestreo que hemos utilizado ha sido el muestreo no probabilístico causal o accidental, que es aquel en el cual el investigador selecciona directa e intencionalmente la muestra, debido fundamentalmente a que tiene fácil acceso a la misma y es representativa de la población (Gil, Rodríguez y García, 1995; Albert, 2006; Sabariego, 2004).

\section{Instrumentos}

Se aplicó una entrevista a los empleadores y empleadoras de investigadores o $\mathrm{PhD}$, para identificar la percepción sobre el quehacer de un graduado o graduada en un programa de doctorado en estudios interculturales, entre las preguntas se destacan: ¿En su organización son necesarios los servicios de uno o más de un Doctor?, ¿En qué áreas o cargos de su organización se puede desempeñar?, ¿Qué funciones podría desempeñar?, ¿Qué tareas debe desarrollar? y ¿Qué habilidades, actitudes, valores y conocimientos debe tener para realizar eficientemente esas tareas y funciones?

También, se ha aplicado una entrevista estructurada que ha permitido recolectar información cualitativa y cuantitativa en relación con el programa académico de Doctorado en Estudios Interculturales. Los participantes tenían que indicar su grado de satisfacción con las afirmaciones propuesta por medio de un "si o un no", pero además de configurar una respuesta a las preguntas siguientes: ¿Ha pensado usted realizar estudios de Doctorado?; ¿Le motiva a usted estudiar un Doctorado en Estudios Interculturales?; ¿Cree usted que estudiar un Doctorado en Innovación en Estudios Interculturales le ayudaría a fortalecer sus conocimientos, saberes y prácticas profesionales en el contexto de la Costa Caribe de Nicaragua y Ab'ya Yala?; ¿En qué modalidad prefiere usted realizar estudios doctorales?; ¿ ¿Qué forma de pago prefiere?

Finalmente, para el análisis documental se construyó una guía de revisión documental para interpretar los significados de contenidos de libros de textos, artículos científicos, artículos de prensa, leyes, currículos, programas de formación y normas institucionales. 


\section{Procedimiento de administración y enfoque ético}

La administración de la entrevista se realizó por parte de los autores durante el año académico 2018. Tenía un carácter anónimo y fue complementado por los sujetos participantes con ayuda de las secretarías académicas de la URACCAN. Con anterioridad a la toma de los datos, se obtuvo tanto el consentimiento libre, previo e informado de los participantes, así como la autorización de las autoridades universitarias.

\section{Análisis y comprensión de los datos}

Para satisfacer los objetivos del estudio se procedió al desarrollo de distintos análisis, primero se ha empleado una matriz de categorización y clasificación de las respuestas, esto ayudó a comprender las respuestas de los participantes. Así mismo, se utilizó Paquete Estadístico para las Ciencias Sociales SPSS v.24, para cuantificar las respuestas de los entrevistados y posteriormente, explicar descriptivamente los resultados. También, se revisaron los textos, documentos, informes, artículos científicos y periodísticos que coadyuvaran al contexto y tendencia del estudio de pertinencia y factibilidad.

\section{Resultados y discusión}

\section{Contexto y tendencia sobre el empleo de investigadores en Nicaragua}

El Estado de Nicaragua es el sexto país de América Latina y el Caribe en dedicar porcentualmente más del Producto Interno Bruto (PIB) a la Educación Superior (1.14\%.) Sin embargo, esta inversión sigue sin permitir que exista un salto en la producción científica. Aunque Cuba es el país de América Latina y el Caribe con mayor índice en el desarrollo de la educación con un 13\% del Producto Interno Bruto (UNESCO, 2013). El informe de Ciencia emitido por la Unesco (2016) señala que Nicaragua incrementó el número de publicaciones científicas de 39 a 54 marcando una tendencia latinoamericana que pasó del $4 \%$ a publicar a el 5.2\% del material científico global, en el período 20052014, aunque sólo hay 9 de estos estudios por cada millón de habitantes. En cambio, Chile despunta esta estadística en América del Sur con 350 por millón. No obstante, Nicaragua, está por encima de sus vecinos: El Salvador (7); Guatemala (6); y Honduras (4), pero muy por debajo de Costa Rica (96) en materia de producción científica. 


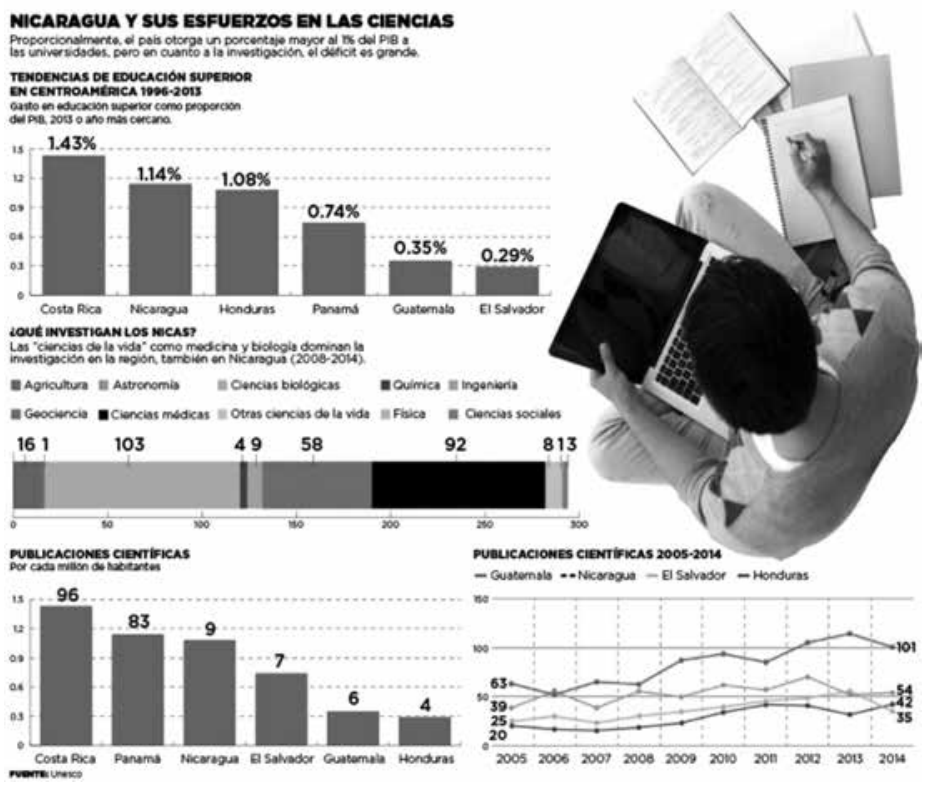

Figura 2: Nicaragua y sus esfuerzos en las Ciencias. Fuente: El Nuevo Diario 2016.

Se puede pensar que hay una mejoría en las publicaciones científicas de Nicaragua para el período 2005-2014, pero en otros años ha tenido mejor desempeño, por ejemplo, la UNESCO (2013) informó que en otros períodos se ha llegado a tener cerca de 100 publicaciones anuales, es decir, el doble de lo actual. Asimismo, destaca que las llamadas ciencias de la vida relacionadas a la medicina y biología representan el mayor número de investigaciones entre 2008-2014 con un total de 203 estudios efectuados. Sin embargo, estos aportes contribuyen muy poco a la economía, por ejemplo, los productos manufacturados no alcanzan el 1\%, mientras que Costa Rica concentra el 43.3\% en este mismo sector, Guatemala y El Salvador están en el rango del 4 y 5 por ciento.

Esto se explica debido al imperceptible número de investigadores e investigadoras. Nicaragua, por ejemplo, es uno de los países con menos científicos en América Latina y el Caribe, muy por debajo de Costa Rica, que supera el promedio latinoamericano con 1,300 investigadores por millón de habitantes. Esta situación obedece a la falta de formación especializada en el grado de Doctor, aunque existe programas de doctorado en el país, los egresados ejercen su tiempo a la docencia, gestión y extensión.

En el contexto del empleo en Nicaragua, los registros administrativos del Instituto Nacional de Seguridad Social (INSS) indican que, en mayo 2017, la afiliación se ubicó en 919 mil 770 trabajadores, con un aumento interanual del 7.1\%. Las actividades económicas con mayor incremento fueron: minas y canteras (15.3\%); comercio, hoteles y restaurantes (14.3\%); agropecuario, silvicultura, caza y pesca (11.9\%); electricidad, gas y agua (8.9\%) y el sector de construcción (8.4\%). En el lapso 2006 a 2017 se observó que 
el salario promedio nominal de los afiliados se situó en $C \$ 9,837,10$ córdobas, con un crecimiento interanual de $5.7 \%$. El salario promedio real mostró un incremento interanual de 2.7\%, y se ubicó en $C \$ 4.616$,6o córdobas (Banco Central de Nicaragua, 2017).

\section{Expectativas de los empleadores sobre las competencias de los futuros doctores en estudios interculturales}

Según empleadores, los servicios del personal con formación doctoral en estudios interculturales son necesarios porque hoy en día la sociedad ha adoptado situaciones cambiantes de manera vertiginosa y la gran mayoría de la población de Nicaragua y específicamente de la Costa Caribe nicaragüense no está asimilando estos cambios y por lo general se aprende a "sobrevivir". En muchas ocasiones se copia algunos modelos de otras sociedades de los cuales se aprende mucho, pero como es natural, no siempre atienden la particularidad de las necesidades sociales, culturales y lingüísticas de nuestras propias sociedades. Además, podemos reconocer que existen cualidades creativas e ingeniosas en los profesionales, pero debemos volverlas más beneficiosas para las comunidades indígenas, afrodescendientes y mestizas de la Costa Caribe. Este acercamiento a la comprensión de la realidad, es lo que nos lleva a la "urgente necesidad de los servicios de uno o más profesionales con el grado de Doctor o PhD", para que gestionen proyectos y programas para los procesos de creación y recreación de conocimientos, saberes y prácticas del país desde la innovación y creatividad de las investigaciones propias.

Con respecto a las áreas de desempeño los entrevistados (mujeres y hombres) coincidieron que puede ser en el ámbito de la investigación sobre Educación Superior que ha establecido el Consejo Nacional de Evaluación y Acreditación (CNEA) en su plan de trabajo y según mandato de la ley 704. También, se requieren personas con nivel de doctorado que puedan desempeñarse como pares académicos. Desde el ámbito del Sistema Educativo Autonómico Regional (SEAR), es necesario que sean docentes investigadores que acompañen a la comunidad y a la formación de hombres y mujeres con pertinencia y calidad. Así mismo, pueden ocupar cargos como: directivos en los distintos subsistemas educativos y administrativos, coordinadores de investigación; gerente de proyectos integrales de desarrollo que acompañen los procesos de autogestión de las comunidades indígenas, afrodescendientes y mestizas de la Costa Caribe de Nicaragua.

Asimismo es importante destacar que pueden realizarse en diversas funciones, entre ellas, como investigador en diferentes ámbitos de la Educación Superior en contextos pluriculturales; gestor de proyectos y programas de investigación, desarrollo e innovación que den respuesta a problemáticas propias; pares académicos en procesos de evaluación y acreditación en el contexto de universidades comunitarias interculturales de Nicaragua y Ab'ya Yala; en la creación y recreación de instrumentos metodológicos para los procesos de evaluación y acreditación en contextos multiétnicos 


\section{EDUCACIÓN}

y compartiendo con el profesorado en el diseño e implementación de metodologías innovadoras propias de los pueblos.

Cabe señalar que desde el punto de vista de la evaluación y acreditación universitaria se espera que un profesional con el grado de Doctor debe desarrollar tareas como:

Investigación sobre diversos ámbitos de Educación Superior. En particular, sobre los contextos educativos pluriculturales existentes en la Costa Caribe de Nicaragua; sobre los modelos educativos y evaluativos interculturales que delinean el accionar de las universidades con modelos educativos propios.

a. Definición de indicadores y estándares de calidad para la evaluación y acreditación de la Educación Superior comunitaria intercultural.

b. Participación en procesos de evaluación y acreditación en universidades interculturales.

c. Desarrollo de instrumentos metodológicos para los procesos de evaluación y acreditación en el contexto pluricultural.

d. Creación y recreación de conocimientos, saberes y prácticas de los pueblos indígenas, afrodescendientes y mestizos.

e. Investigación de las problemáticas y las buenas prácticas que se manifiestan en la sociedad de la Costa Caribe nicaragüense.

Desde la perspectiva del SEAR el profesional con grado de Doctor ( $\mathrm{PhD}$ ) debe realizar tareas como: investigación, docencia, asesoría, planes de vida, sistematizaciones y socialización de procesos de creación y recreación de conocimientos, saberes y prácticas.

El profesional graduado en un programa de Doctorado en Estudios Interculturales tiene que poseer conocimientos y habilidades de comunicación, trabajo en equipo, lectura comprensiva, dominio de segunda lengua, dominio de las TIC, entre los más relevantes. Perspectiva estratégica, capacidad de análisis y sentido común, debe saber planificar y organizar cualquier tipo de actividad, capacidad de toma de decisiones, ser innovador y emprendedor. Actitudes: Disposición al trabajo, disciplina, responsabilidad, compromiso con la educación, ser asertivo, autonomía, creatividad, motivador. Valores: Respeto, ética, solidaridad, sororidad, equitativo, empatía, generosidad, integridad, honestidad, tolerancia, humanista y responsable.

Los futuros doctores deben estar formados en diversas líneas de investigación asociadas a una gran área del conocimiento: La Interculturalidad con perspectiva de género y visión holística del mundo con sus diferentes sistemas de conocimientos. Con aptitudes y actitudes para mantenerse actualizado en los conocimientos, especializados de su área de investigación y abierto al diálogo de saberes en equidad epistémica. Potencian su creatividad, innovación, emprendedurismo, desarrollen el pensamiento crítico, actualicen y profundicen sus conocimientos y encuentren las 
formas de presentación adecuadas de manera oral y escrita para evidenciar la importancia y originalidad del trabajo desarrollado.

\section{Tendencia y demanda de estudios de los profesionales con grado de Maestría}

Se ha consultado a los participantes con la pregunta: ${ }_{i}$ Ha pensado usted realizar estudios de Doctorado?, estos han contestado:

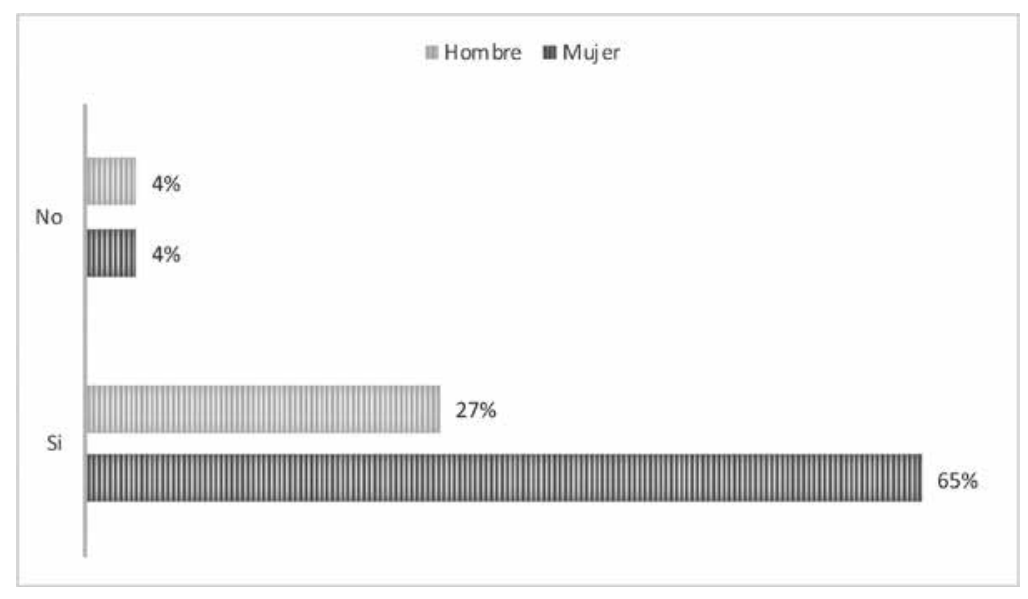

Figura 1: ¿Has pensado usted realizar estudios de doctorado?.

El 92\% de los entrevistado afirmaron que sí han pensado estudiar un doctorado, porque:

el nuevo contexto de la era del conocimiento y el mundo cambiante y acelerado que actualmente vivimos exigen profesionales mejor cualificados con conocimientos holísticos, integrales con una visión amplia de las cosas para poder aportar y asumir los retos actuales con pertinencia cultural que aporten al buen vivir de los pueblos.

También, las personas entrevistadas afirmaron que el estudiar un doctorado ayuda al crecimiento profesional, además, que mejora la calidad de las competencias en la docencia e investigación garantizando la pertinencia de los aprendizajes, contribuyendo a comprender los escenarios que viven los pueblos de la Costa Caribe nicaragüense. 


\section{EDUCACIÓN}

Cuadro 2: Piensas en realizar estudios de doctorado

- Formación profesional

- Garantizar la pertinencia del aprendizaje

- Actualización de conocimientos

- Acreditación y calidad profesional

- Crecer profesionalmente

- Competencias para la globalización

- Actualización ante las nuevas realidades

- Impulsar procesos académicos e investigativos

- Aportar a un mundo más equitativo

- Retos y compromisos sociales

- Atención con calidad

- Mejor desempeño institucional

¿Han pensado usted realizar estudios de doctorado?
- Comprender los escenarios que viven los pueblos

- Aportar al desarrollo educativo

- Sensibilización a las realidades de los pueblos

El 8\% restantes afirmaron que no pueden realizar estudios de Doctorado por problemas de salud y estar cerca de su jubilación; así mismo, expresan que no están ejerciendo la docencia y los gastos económicos que generaría iniciar un proceso de formación a nivel de doctorado son muy altos.

Se consultó a los participantes, ¿sobre la motivación de estudiar un programa de Doctorado en Estudios Interculturales?, sus respuestas son:

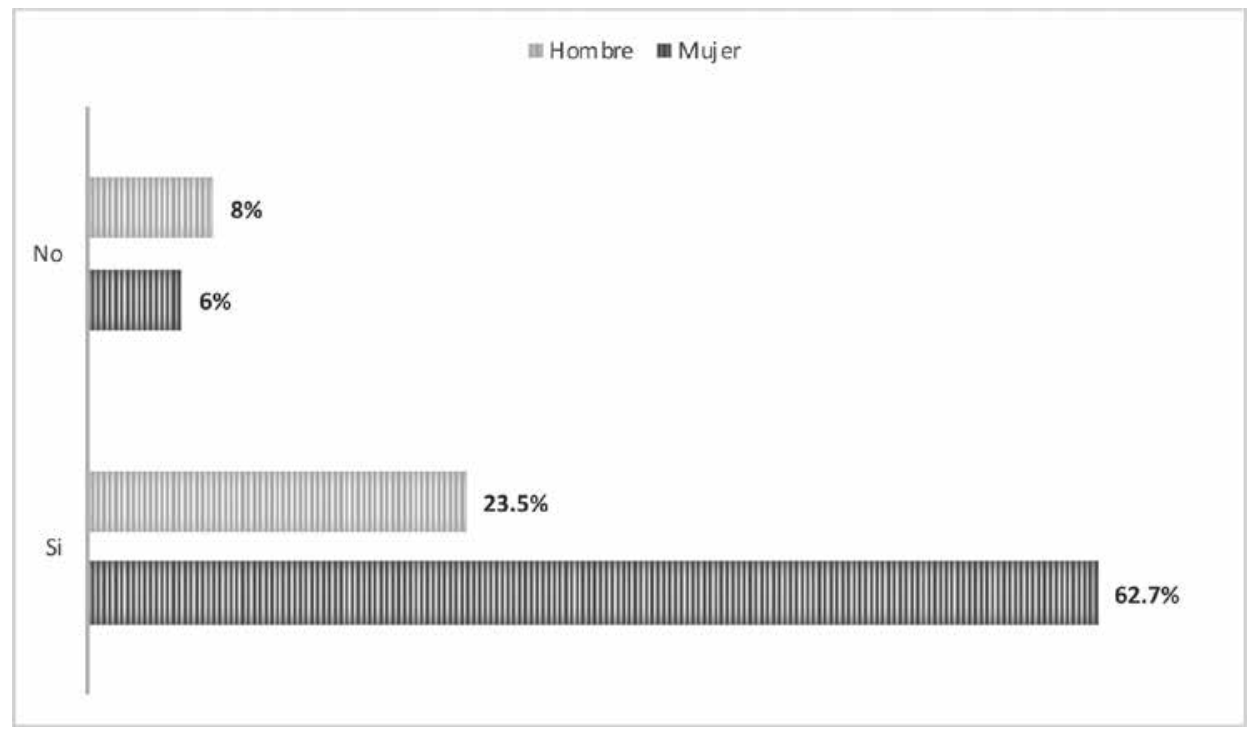

Figura 2: Le motiva a usted estudiar un Doctorado en Estudios Interculturales. 
El 86\% de los participantes les motiva estudiar un programa doctoral en estudios interculturales afirmando que "viene a resolver una demanda de hombres y mujeres con alto nivel cualificado de formación, además, que los doctores con una formación contextualizada le da el plus que contextos pluriculturales requieren para ser más pertinentes en los estudios, investigaciones y propuestas con planteamientos que consideren los desafíos de la diversidad cultural sus razonamientos, explicaciones, comportamientos y alternativas de mejora en el marco de las Autonomías de los Pueblos.

Cuadro 3: Motivación hacia el estudio de programa de doctorado en innovación en estudios interculturales

- Alto nivel cualificado de formación

- Fortalecer los procesos de comunicación intercultural

- estrategias que fundamenta el Buen Vivir y el Vivir Bien de nuestros Pueblos

- Para comprender las realidades interculturales

- Gestionar la Docencia

- Para entender y comprender escenarios ricos en diversidad cultural y étnica

- Para promover la calidad e innovación al contexto que estamos desarrollando la educación con enfoque intercultural.

- Es parte de la gestión de la filosofía institucional de URACCAN

\begin{tabular}{|c|c|}
\hline $\begin{array}{l}\text { ¿Le motiva a usted es- } \\
\text { tudiar un Doctorado } \\
\text { en Estudios } \\
\text { Interculturales? }\end{array}$ & $\begin{array}{l}\text { - Más pertinentes las investi- } \\
\text { gaciones. } \\
\text { - Para investigar desde el } \\
\text { enfoque intercultural. } \\
\text { - } \text { Desarrollar políticas inter- } \\
\text { culturales para los pueblos } \\
\text { - Metodologías y herramien- } \\
\text { tas para acompañar a los } \\
\text { pueblos. } \\
\text { - Mejorar las convivencias de } \\
\text { todos los pueblos y comu- } \\
\text { nidades } \\
\text { - Crear convivencias armó- } \\
\text { nicas, relaciones intercultu- } \\
\text { rales exitosas entre todos y } \\
\text { todas. } \\
\text { - Mejorar las condiciones de } \\
\text { vida } \\
\text { - apuntar hacia el empren- } \\
\text { dedurismo e innovación } \\
\text { empresarial. } \\
\text { Gestionar la investigación } \\
\text { desde y para la intercultura- } \\
\text { lidad de los pueblos. }\end{array}$ \\
\hline
\end{tabular}

También, los participantes comentaron que el doctorado contribuirá a mejorar las condiciones de vida de las personas partiendo de la comprensión de cómo viven, su cultura, su forma de ver el mundo, y hasta entonces juntos plantearían soluciones a los problemas de las comunidades desde cada uno de los campos de acción. Asimismo, estudiar un programa académico con este perfil contribuye a desarrollar escenarios investigativos interculturales que apuntan hacia el emprendedurismo e innovación para el desarrollo con identidad. Mientras que el 14\% afirmó que no le motiva estudiar 
un Doctorado en Estudios Interculturales porque su perfil profesional no se vincula con el programa doctoral, igualmente les gustaría profundizar en estudios de carácter profesional en sus líneas de especialización y formación de postgrado.

Por otra parte, el 98\% de los entrevistados afirmaron que el programa de Doctorado en Estudios Interculturales les ayudará a fortalecer sus conocimientos, saberes y prácticas profesionales en el contexto de la Costa Caribe nicaragüense y Ab'ya Yala, además que les permitirá tener una visión más holística del mundo real en que vivimos y actuamos, así como adquirir habilidades y herramientas para investigar y hacer planteamiento más coherente con visión intercultural, respetando a la madre tierra e implementando prácticas innovadoras coherentes e inclusivas a las comunidades.

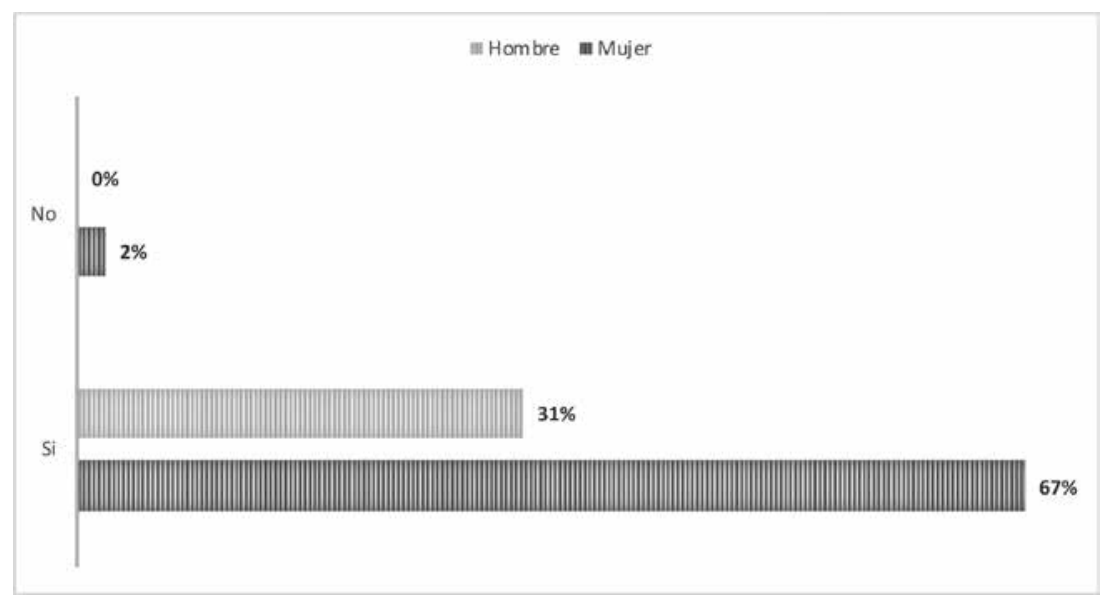

Figura 3: Fortalecer conocimientos, saberes y prácticas para el contexto de la CCN y Ab'ya Yala.

Igualmente, argumentaron que es una oportunidad para conocer a profundidad el contexto de la Costa Caribe de Nicaragua y Ab'ya Yala, es decir, y entender la diversidad cultural de los pueblos indígenas, afrodescendientes y mestizos. Comprender el desarrollo de sus formas de investigación propias que ayudaría a llevar conocimientos, saberes y prácticas a las aulas de clases. Finalmente, aportaría al crecimiento profesional y el desarrollo con identidad de las comunidades desde sus propias cosmovisiones, así como al fortalecimiento institucional de la URACCAN. Sin embargo, el 2\% de los participantes afirmaron que no, por el desconocimiento del perfil del programa de Doctorado. 
Cuadro 4: Conocimientos, saberes y prácticas fortalecidas

- Consolidar el Modelo de Universidad Comunitaria Intercultural.

- Construcción colectiva de conocimientos interdisciplinarios.

- Investigaciones sobre la interculturalidad y generar mayores aportes a nuestra universidad.

- Entender, comprender, convivir y trabajar con estudiantes en un ambiente intercultural tomando en cuenta las prácticas y saberes en el contexto de la costa caribe.

- Generar investigaciones para la gestión de proyectos $1+D+1$.
Conocimientos, saberes y prácticas profesionales en el contexto de la Costa Caribe nicaragüense y Abya Yala
- Visión más holística del mundo real.

- Comprender el conflicto y las manifestaciones que se suscitan en nuestro contexto de diversidad cultural.

- Enriquecería las nuevas prácticas y saberes ancestrales y daría valor a la cosmovisión de nuestros pueblos.

- Formación de profesionales conscientes de su realidad.

- Permitirá contribuir a la mejora de los múltiples hilos sociales, políticos, culturales, lingüísticos de nuestra Costa Caribe.

- Entender la identidad de los pueblos.

Asimismo, se consultó sobre la modalidad de estudios preferidos por los aspirantes, a lo que respondieron lo siguiente:

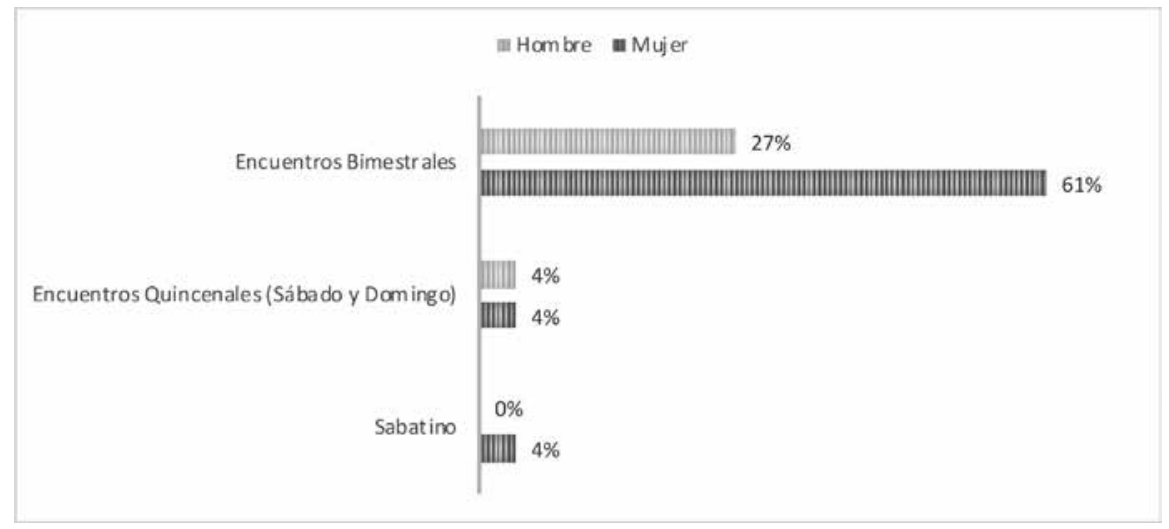

Figura 4: ¿En qué modalidad prefiere usted realizar estudios doctorales?

En la figura 5, podemos observar que el $88 \%$ de los participantes desean estudiar sus estudios doctorales por encuentros bimestrales, un $8 \%$ de los entrevistados desea estudiar por encuentros quincenales (sábado y domingo), y finalmente un $4 \%$ desea estudiar en la modalidad sabatina. Por lo que se infiere que los entrevistados 
consideran idóneo estudiar el programa de Doctorado en Estudios Interculturales en la modalidad de encuentros bimestrales, porque permitiría cumplir con las jornadas laborales y las responsabilidades de estudios.

Sobre las formas de pagos para realizar estudios de doctorados, los y las participantes respondieron lo siguiente:

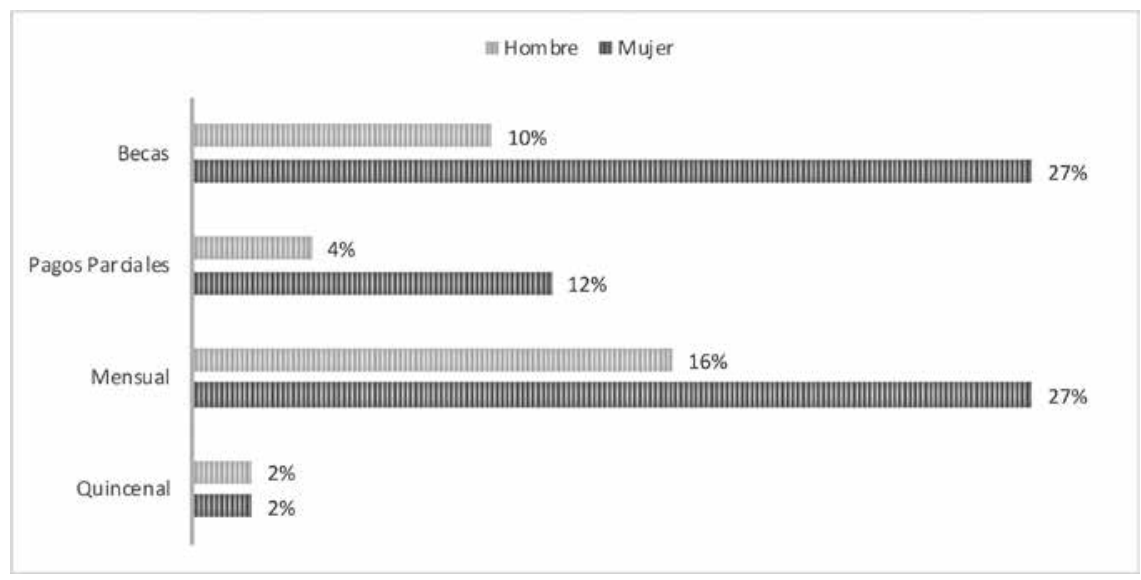

Figura 5: Estaría dispuesto asumir una forma de pago para estudiar el Doctorado en Estudios Interculturales.

$\mathrm{El} 43 \%$ desea realizar sus pagos mensuales, $16 \%$ desea realizar pagos parciales $(50 \%$ al inicio, $25 \%$ a mitad del programa y $25 \%$ antes de su finalización) y el $4 \%$ afirmaron que desean efectuar sus pagos quincenales. Sin embargo, un $37 \%$ de los entrevistados expresan que es necesario un programa de becas para poder llevar a cabo sus estudios doctorales.

\section{Estudio de factibilidad del programa de Doctorado en Estudios Interculturales}

Los estudios de factibilidad se refieren a la capacidad para responder a los requerimientos de los programas académicos (recursos físicos, financieros, materiales y talentos humanos) en relación con la demanda del programa académico de Doctorado en Estudios Interculturales.

\section{Recursos físicos}

La URACCAN tiene presencia en las Regiones Autónomas de la Costa Caribe de Nicaragua. Cuenta con 4 recintos (Bluefields, Bilwi, Nueva Guinea y Las Minas) y 4 extensiones universitarias (Waspam, Rosita, Bonanza y Waslala) localizadas en las principales localidades de las zonas etnolingüísticas de las regiones autónomas. 
Además, de una oficina de gestión en la ciudad de Managua. Asimismo, cuenta con plataformas virtuales; laboratorios de idiomas, computación y naturales; salas de video conferencias; sistema de biblioteca virtuales y repositorio institucional y revistas científicas.

- Plataformas Virtuales: La URACCAN utiliza la educación virtual y a distancias a través de la plataforma Moodle donde se desarrollan las clases, permitiendo el acceso al estudiantado en cualquier parte del mundo y momento.

- Laboratorios de Idiomas y computación: Son espacios de interacción especializados con software específicos instalados, que permiten al estudiantado realizar prácticas en tiempo real, donde el profesorado es el facilitador y el estudiantado es constructor de su propio aprendizaje, es decir, se promueve el aprendizaje autónomo a través de situaciones prácticas.

- Salas de video conferencia: La URACCAN cuenta con dos sistemas de video conferencia, una plataforma web y un sistema de interconexión por IP (Protocolo de Internet), estas actualmente se utilizan para conferencias magistrales y procesos de enseñanza-aprendizaje.

- Sistema de bibliotecas virtuales y repositorio institucional: Es un lugar físico y virtual con espacios de estudios adecuados y recursos de información necesario para estudiar e investigar, además con tecnologías de la información y comunicación para el trabajo de proyectos de investigación y la búsqueda de literatura científica en bases de datos nacionales e internacionales.

- Revistas Científicas: La URACCAN posee dos revistas científicas indexadas en plataformas nacionales e internacionales. Tienen como objetivo la publicación de artículos inéditos y de revisión crítica que aportan en diversas áreas del conocimiento, saberes y prácticas. Además, las revistas cumplen los requisitos de la Latindex.

- Laboratorios Naturales: Espacios vivos donde se construyen y recrean conocimientos, saberes y prácticas en armonía con la Madre Tierra; que contribuyen a la revitalización de la identidad y cultura de los pueblos de la Costa Caribe mediante la innovación, emprendimiento, producción y comercialización fundamentado en los lineamientos, principios y valores institucionales para el Buen Vivir (Hernández et al, 2016).

- Laboratorios de comunicación intercultural: Una red de radios y programas de TV-cable de educación comunitaria que son espacios para el diálogo y la comunicación intercultural donde interactúa la comunidad universitaria y los pueblos a través de sus prácticas didácticas y pedagógicas propias.

- Aulas Interculturales: Las aulas representan ambientes sencillos y amigables donde los estudiantes y docentes de diferentes pueblos comparten la diversidad de sus voces, lenguas, conocimientos, saberes y prácticas sobre sus formas de vida y maneras de ver el mundo en un marco de complementariedad, 
diálogo intercultural y de género (URACCAN, 2014b, p. 16). Además, son el espacio de diálogo para la creación, recreación, diseminación e intercambio de conocimientos, saberes y prácticas a través de procesos de participación democráticos e inclusivos donde interactúan los conocimientos propios de los pueblos en un ambiente de aprendizaje con incorporación de las tecnologías del aprendizaje (Flores, 2017).

También, la URACCAN cuenta con un Plan Estratégico Institucional (2015-2019) y un marco normativo institucional que enfatiza políticas, normas, reglamentos y guía orientadoras del quehacer institucional. Entre las políticas se puede mencionar: Política institucional de investigación; Política de extensión social y comunitaria; Política intercultural de género; Política de comunicación intercultural; Políticas de administración de recursos humanos y Política de gestión de la cooperación externa

- Documentos normativos: Normativa de propiedad intelectual; Normativa de actos de graduación; Normativa fondo especial de desarrollo y sostenibilidad de URACCAN; Normativa de carga horaria docente de tiempo completo; Normativa para la aplicación de exámenes de suficiencia; Normativas para tutorías de asignaturas; y Normativa de la carga horaria y del pago de docencia del personal docente y administrativo. Reglamentos: Reglamento de Régimen Académico, Reglamento de bibliotecas, Reglamento docente, Reglamento electoral, Reglamento estudiantil, Reglamento de becas, Reglamento interno, Reglamento de postgrado y educación continua, Reglamento del personal administrativo, Reglamento de pasantía académica estudiantil, Reglamento de becas para funcionarios de URACCAN y Reglamento del Consejo Universitario

- Guías orientadoras, documentos académicos, metodológicos, manuales y agendas: Guía de redacción de artículos técnicos; Guía de redacción de artículos científicos, Guía para el diseño y presentación de protocolos de investigación, Modelo Pedagógico de URACCAN, Manual de políticas y procedimientos contables, Marco conceptual y metodológico para evaluar programas académicos, Agenda para el desarrollo con Identidad, Costos y pagos por servicios académicos, Manual contable, y Manual de normas, funcionamiento y organización institucional.

\section{Recursos financieros}

El presupuesto de URACCAN está determinado por 3 fuentes principales: asignación presupuestaria del Presupuesto General de la República vía 6\% asignado a las universidades miembros del CNU, fondos propios recaudados mediante diferentes servicios prestados por la Universidad y financiamiento de proyectos por la Cooperación Externa.

\section{Talentos humanos}

La comunidad universitaria de la URACCAN ha ido creciendo hasta comprender 9,915 estudiantes y un personal de 933 trabajadores, incluyendo 497 trabajadores 
permanentes y 436 horarios. A lo largo de su existencia, la universidad ha graduado a un total de 7,702 profesionales en las diferentes áreas del conocimiento. En el 2017, la URACCAN acumula 13 talentos humanos con el grado de $\mathrm{PhD}$ de los cuales, 2 estudiantes realizaron sus estudios en universidades nacionales y 11 en universidades de América Latina y Europa. Estos profesionales se caracterizan por ser de las áreas siguientes: el 16\% en Humanidades, Ciencias Jurídicas y Sociales; 15\% Ciencia, Tecnología y Medio Ambiente; el 54\% Ciencias de la Educación e Idiomas y un 15\% a Ciencias Económicas y Administrativa. Véase figura 7.

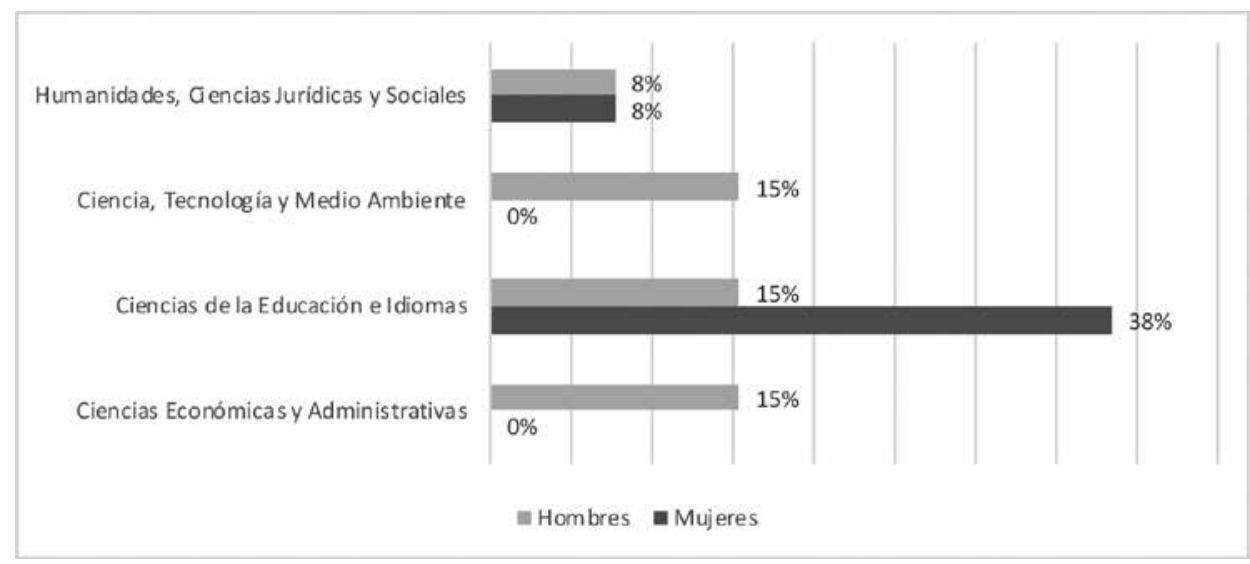

Figura 6: Distribución del personal con grado de PhD de la URACCAN.

También, la URACCAN posee Institutos y Centros de Investigación, que están mandatados a realizar investigaciones según las líneas de especialización, entre ellos: Instituto de Recursos Naturales, Medio Ambiente y Desarrollo Sostenible. (IREMADES), Instituto de Promoción e Investigación Lingüística y Cultural. (IPILC), Instituto de Medicina Tradicional y Desarrollo Comunitario. (IMTRADEC), Institutos de Estudios y Promoción de la Autonomía. (IEAPA), Centro de Estudios e Información de la Mujer Multiétnica (CEIMM), y Centro de Información Socio-Ambiental (CISA). Entre los principales temas que se abordan desde los Institutos y Centros de Investigación encontramos: 1) Cambio Climático, 2) Soberanía Alimentaria, 3) Biodiversidad, 4) Industrias extractivas o megaproyectos, 5) Salud Intercultural, 6) Educación Intercultural Bilingüe, 7) Relaciones Interculturales y Ciudadanía Intercultural, 8) Autonomía Regional, 9) Desarrollo con Identidad, 10) Enfoque de Género, 11) Violencia de Género, 12) Procesos de integración económica, política, social y cultural, 13) Práctica de la medicina tradicional, entre otros (URACCAN, 2017a).

\section{Conclusiones}

Esta investigación ha analizado la pertinencia y factibilidad de un programa de Doctorado en Estudios Interculturales. Resulta relevante estudiar la pertinencia, ya que permite estar en contacto con las políticas, con el mundo del trabajo, con los 


\section{EDUCACIÓN}

demás niveles del sistema educativo, con la cultura y las culturas, con el estudiantado y profesorado, con todos, siempre y en todas partes (Tünnermann, 2006, p. 7). Así como, estudiar la factibilidad, porque consiste en la articulación de un conjunto de actividades vinculadas entre sí, cuya ejecución permitirá él logró de objetivos previamente definidos en atención a las necesidades que pueda tener una institución o grupo social en un momento determinado.

Desde esta perspectiva, se encontró que la pertinencia y factibilidad del programa de doctorado se resume de la manera siguiente:

Existe una necesidad en la formación de investigadores de alto nivel que contribuyan a la creación y recreación de conocimientos, saberes y prácticas innovadoras y emprendedoras, teniendo en cuenta que el conocimiento es un derecho humano universal, un bien público social y común para la soberanía, buen vivir y emancipación de los pueblos, y para la construcción de la ciudadanía latinoamericana y caribeña, retomando, además, la necesidad de acceso libre a los conocimientos y la importancia de la inversión científica como un deber de cada Estado (UNESCO, 2017).

La expectativa de los empleadores sobre las competencias de los futuros doctores en Estudios Interculturales se puntualiza en que deben ser competentes para la gestión de programas y proyectos para la generación de investigación, desarrollo e innovación, además, definir indicadores y estándares de calidad para evaluación y acreditación universitaria. Asimismo, responder a los roles de la educación intercultural y diversidad de las poblaciones de América Latina y el Caribe. En este sentido, es necesario fortalecer las competencias de investigación en el campo de la innovación en estudios interculturales con fundamentos, conocimientos, herramientas teóricas, prácticas y metodológicas para el análisis de realidades desde las cosmovisiones de los pueblos, pensamiento crítico, diálogo de saberes y haceres, con perspectiva intercultural de género que conlleven al Buen Vivir (URACCAN, 2017b).

El 92\% de los participantes en la investigación desean realizar estudios de doctorado, vinculado a estudios interculturales, ya que les ayudará a su crecimiento profesional, mejorará la calidad de las competencias en la docencia y contribuirá a comprender los escenarios que viven los pueblos de la Costa Caribe de Nicaragua. Es decir, formar hombres y mujeres con conocimientos, saberes, capacidades, valores, principios, actitudes humanistas, sentido de emprendimiento e innovación en equilibrio y armonía con la Madre Tierra para el fortalecimiento de las autonomías de los pueblos (URACCAN, 2014a).

Con relación a la factibilidad del programa de doctorado en Estudios Interculturales desde la perspectiva de recursos físicos, recursos financieros y talentos humanos. En este sentido, los recursos físicos destacados son: plataformas virtuales, laboratorios de idiomas y computación, salas de video conferencia, sistema de bibliotecas virtuales 
y repositorio institucional, revistas científicas, laboratorios naturales, laboratorios de comunicación intercultural y aulas interculturales. Además, se cuenta con políticas, normativas, reglamentos, guías orientadoras, documentos académicos, metodológicos, manuales y agendas institucionales (URACCAN, 2016). En los recursos financieros se destaca que el presupuesto universitario está constituido por tres fuentes: Fondos propios, fondos de cooperación y fondos del $6 \%$ constitucional. Sobre los talentos humanos, es importante destacar que la universidad tiene 13 hombres y mujeres formados con el grado de doctor, además, que la implementación de acciones de acompañamiento intercultural lo realiza desde los siete centros e institutos de investigación.

A manera de conclusiones, existe demandas de formación especializada en investigación para mujeres y hombres de la Costa Caribe nicaragüense. Es decir, existe la necesidad de formación de investigadores con el nivel de doctorado con conocimientos, habilidades, actitudes y valores para realizar aportaciones de relevancia teórica, metodológica y analítica para la academia y la sociedad en general con perspectiva intercultural de género, que coadyuven de manera innovadora a la revitalización de conocimientos, saberes y prácticas, así como la reflexión y solución de los problemas sociales, políticos, económicos, ambientales y multiculturales de los pueblos; desarrollar investigaciones con perspectiva intercultural de género mediante el análisis crítico y el diálogo de saberes; comunicar desde una perspectiva intercultural de género conocimientos y resultados de investigaciones individuales o colectivas en congresos, foros, simposios, seminarios, libros y revistas de reconocimiento en las disciplinas sociales y humanísticas; $y$, finalmente gestionar programas y proyectos de investigación desarrollo e Innovación con perspectiva intercultural de género (URACCAN, 2017b).

\section{Lista de referencias}

Abreu, L., Cruz, V., \& Martos, F. (2014). Evaluación de programas de postgrados: Guía de Autoevaluación. España: AUIP.

Arrieta de Meza, B., \& Meza Cepeda, R. D. (2014). Factibilidad de un programa de doctorado en lingüística para la universidad del zulia. Orbis. Revista Científica Ciencias Humanas, 10(29), 155-173.

Banco Central de Nicaragua "BCN”. (2017). Informe de desempeño de la economía nacional y perspectivas. Managua: $\mathrm{BCN}$.

Castillo, L., Ruíz, L., Peralta, M., Dávila, A., Simons, I., Perera, F. (2013). Oferta académica de la URACCAN en el Nivel de grado para el quinquenio 2014-2018. Ciencia e Interculturalidad, 13 (2), 47-62. DOI: http://dx.doi.org/10.5377/rci.v13i2.1276

Flores, W., O. (2017). Sistema de Evaluación de los Aprendizajes desde la Pedagogía Intercultural. Managua: URACCAN. 


\section{EDUCACIÓN}

García-Guadilla, C. (1997). El valor de la pertinencia en las dinámicas de transformación de la educación superior en América Latina. En: La educación superior en el Siglo XXI. Visión de América Latina y el Caribe. Tomo I. Colección Respuestas. Caracas: Ediciones CRESALC/UNESCO.

Hernández, O., Díaz, D., Ruiz, A., Ramírez, B., Gutiérrez, G., Morales, N. (2016). Los laboratorios naturales un espacio vivo para la formación integral del estudiantado de la Universidad Comunitaria Intercultural. Revista Universitaria del Caribe, 16(1), 66-75. DOI: http://dx.doi.org/10.5377/ruc.v16i1.3231

Sobrinho, J., Stubrin, A., Martín, E., González, L. E., Espinoza, O., \& Goergen, P. (2008). Calidad, pertinencia y responsabilidad social de la universidad latinoamericana y caribeña. Tendencias de la educación superior en América Latina, 61, 87-112.

Tünnermann, C. (2006). Pertinencia y calidad de la educación superior. Lección inaugural efectuado en Guatemala. Recuperado el 21 de febrero de 2018 en: http://biblio2. url.edu.gt:8991/libros/leccion\%2oinaugural20o6texto.pdf

UNESCO. (1998). La Educación Superior en el umbral del siglo XXI. Caracas: IESALC-UNESCO.

UNESCO. (2017). Declaración de Quito sobre la ciencia, los conocimientos, las tecnologías $y$ las artes. Recuperado el 21 de febrero de 2018 en: http://observasur.org/

URACCAN. (2014a). Plan Estratégico Institucional 2015-2019 de la Universidad de las Regiones Autónomas de la Costa Caribe Nicaragüense. Managua: URACCAN.

URACCAN. (2014b). Primer Informe de Evaluación Anual. Plan de Mejora de URACCAN 2015-2018. Managua: URACCAN.

URACCAN. (2016). Proyecto Educativo Institucional de la Universidad de las Regiones Autónomas de la Costa Caribe Nicaragüense. Managua: URACCAN.

URACCAN. (2017a). Perfil Institucional de la Universidad de las Regiones Autónomas de las Regiones Autónomas de la Costa Caribe Nicaragüense. Managua, URACCAN.

URACCAN. (2017b). Currículo del programa de Doctorado en Estudios Interculturales. Managua, URACCAN. 